TAVARES, P. A.; RAMOS, M. C.; PEÇANHA, A. da S. Aplicação das sete ferramentas da qualidade em uma empresa de recapagem de pneus no centro-oeste de Minas Gerais.

\title{
APLICAÇÃO DAS SETE FERRAMENTAS DA QUALIDADE EM UMA EMPRESA DE RECAPAGEM DE PNEUS NO CENTRO-OESTE DE MINAS GERAIS
}

Priscilla Angélica Tavares

Egressa do Curso de Engenharia de Produção do UNIFOR-MG

Marcelo Carvalho Ramos

Professor do UNIFOR-MG

Andréa da Silva Peçanha

Professora do UNIFOR-MG

Recebido em: 29/09/2013

Aprovado em: 29/11/2013

\section{RESUMO}

Em um mercado competitivo, em que os clientes estão cada vez mais exigentes, principalmente em relação à qualidade dos produtos e serviços, as empresas precisam buscar técnicas de gestão e ferramentas para a qualidade contínua. Neste contexto, o presente trabalho apresenta a aplicação das sete ferramentas da qualidade para análise de falhas no processo produtivo de uma recapagem de pneus, localizada no Centro-Oeste de Minas Gerais. Foi realizada uma análise detalhada no processo de recapagem, desde a chegada do pneu à empresa até à inspeção final, em que foram utilizadas as ferramentas da qualidade, para avaliação das etapas do processo que apresentaram falhas, sendo assim propostas ações para melhorias no processo produtivo da respectiva empresa.

Palavras-chave: Qualidade. Produtividade. Ferramentas da qualidade. Recapagem.

\section{IMPLEMENTATION OF THE SEVEN QUALITY TOOLS IN A TIRE RETREADING COMPANY IN WEST CENTRAL MINAS GERAIS}

\begin{abstract}
In a competitive market where customers are increasingly demanding, especially in terms of quality of products and services, companies must seek management techniques and tools for continuous quality. In this context, this work presents the application of the seven quality tools for analysis of failures in the productions process of retread tire located in the west central of Minas Gerais. We performed a detailed analysis of the process of retreading, since the arrival of the tire in the company until the final inspection, where we used the quality tools for evaluation of process steps that failed, and consequently been proposed actions for process improvements.
\end{abstract}

Keywords: Quality. Productivity. Quality tools. Retreading. 
TAVARES, P. A.; RAMOS, M. C.; PEÇANHA, A. da S. Aplicação das sete ferramentas da qualidade em uma empresa de recapagem de pneus no centro-oeste de Minas Gerais.

\section{INTRODUÇÃO}

Dentro de um cenário mundial em que predomina a competição, os clientes estão cada vez mais exigentes e conscientes de seus direitos. A preocupação com a qualidade tornou-se fator essencial para a sobrevivência das empresas no mercado. Elas necessitam aperfeiçoar seus processos, produtos e serviços, por isso buscam técnicas de gestão, para obter um melhor gerenciamento dos recursos, visando à maior lucratividade e fidelidade de seus clientes.

As empresas precisam focar em seus processos, no intuito de verificar se os mesmos possuem falhas que elevam o custo final do produto, reduzem a produção ou comprometem a qualidade. Existem várias ferramentas que auxiliam os gestores nessa análise, cabe a cada um analisar aquelas que melhor se adaptam à realidade e à necessidade de suas organizações.

Dentre essas ferramentas, existem os programas e ferramentas da qualidade que são recursos que contribuem para a solução de problemas e auxiliam na tomada de decisão. Segundo Alvarez (2001), as sete ferramentas da qualidade formam um conjunto de ferramentas estatísticas que são utilizadas pelas organizações, com o objetivo de melhoria da qualidade dos produtos, processos e serviços.

Ao analisar a utilização das sete ferramentas da qualidade em um processo de recapagem de pneus, parte-se da importância que esse setor possui no mercado, uma vez que a qualidade do processo é de suma importância para garantir a longevidade de uma empresa que atua em um mercado de extrema competição.

O setor de recapagem possui vantagem econômica, visto que custo de um pneu recauchutado é inferior ao preço de um novo. Possui, também, vantagem ecológica, pois a recapagem prolonga a vida do pneu, e cada pneu recapado é um a menos a ser lançado no meio ambiente.

Após uma análise preliminar do processo, chega-se a uma interrogação: quais são as etapas do processo de recapagem que apresentam maior número de falhas no fluxo de produção colocando em risco a qualidade do serviço?

Este trabalho objetivou analisar as etapas do processo de produção de uma empresa de recapagem de pneus no Centro-Oeste de Minas Gerais, mensurando perdas de matéria prima, controle de desperdícios e causas de retrabalho, por meio da aplicação das sete ferramentas da qualidade, resultando em uma análise do processo como um todo. A partir dessa análise, foi possível propor soluções que minimizem as falhas encontradas, possibilitando elevar o nível de qualidade, diminuindo os custos e, consequentemente, aumentando os lucros e a satisfação do cliente final. 
TAVARES, P. A.; RAMOS, M. C.; PEÇANHA, A. da S. Aplicação das sete ferramentas da qualidade em uma empresa de recapagem de pneus no centro-oeste de Minas Gerais.

\section{REFERENCIAL TEÓRICO}

Para se entender melhor o papel da qualidade dentro das organizações, é preciso compreender sua evolução ao longo da história.

\subsection{Histórico da qualidade}

Segundo Paladini (1995), a história da qualidade iniciou por volta de 1900, quando surgiram os primeiros gráficos de controle, que foram desenvolvidos por W. Shewhart.

Alvarez (2001) descreve que as primeiras ações desenvolvidas no sentido de buscar a qualidade surgiram nos Estados Unidos, em 1920. Em 1924, é formado o departamento de Engenharia de Inspeção pela empresa americana Western Eletric.

Segundo Longo (1996), a partir de 1950 começa a preocupação com a qualidade. Nessa época, a qualidade deixa de ser uma responsabilidade apenas do departamento específico e passa a ser uma preocupação da empresa como um todo. Nos anos 70, com a crise do petróleo, a economia enfraquece e a preocupação das empresas passa a ser com os custos de seus produtos. Alvarez (2001) complementa que é nesse período que surge o controle total da qualidade e os círculos de controle da qualidade, aplicando as sete ferramentas da qualidade.

Conforme Oliveira et al. (2006), a evolução da qualidade teve três fases: era da inspeção, era do controle estatístico e era da qualidade total.

Longo (2006) descreve que, na era da inspeção, o objetivo principal era detectar os defeitos de fabricação e que não existia uma metodologia para realizar essa inspeção. Já na era do controle estatístico, Oliveira et al. (2006) mencionam que o controle de inspeção era feito por seleção aleatória de alguns produtos e, a partir dessa análise, era verificada a qualidade do lote inteiro. Segundo Corrêa e Corrêa (2008), a era da qualidade total é a vivida atualmente. $\mathrm{O}$ ponto central passa a ser o cliente e as organizações concentram seus esforços em atendê-lo.

\subsection{Conceitos e definições de Qualidade}

Para Gaither e Frazier (2007, p. 489) “a qualidade de um produto ou serviço é a percepção do cliente do grau que o produto ou serviço atende as suas expectativas."

Juran e Gryna (1991, p. 11) completam que "a qualidade consiste nas características 
TAVARES, P. A.; RAMOS, M. C.; PEÇANHA, A. da S. Aplicação das sete ferramentas da qualidade em uma empresa de recapagem de pneus no centro-oeste de Minas Gerais.

do produto que vão ao encontro das necessidades dos clientes e dessa forma proporcionam a satisfação em relação ao produto."

Campos (1992, p. 2) menciona que "um produto ou serviço de qualidade é aquele que atende perfeitamente, de forma confiável, de forma acessível, de forma segura e no tempo certo às necessidades do cliente.”

Para Miguel (2001), os métodos para a qualidade são comandados por especialistas, que têm a responsabilidade de apoiar todos os setores de uma empresa.

Segundo Gianesi e Corrêa (1996), os clientes utilizam vários critérios para avaliar a qualidade de um serviço, sendo: flexibilidade, competência, credibilidade/segurança, tangíveis, custo, acesso, atendimento/atmosfera, velocidade de atendimento e consistência.

\subsection{Ferramentas da Qualidade}

Segundo Corrêa e Corrêa (2008), as sete ferramentas da qualidade são: fluxograma ou diagrama de processo; análise de Pareto; diagrama de causa e efeito ou diagrama de Ishikawa; diagrama de dispersão ou correlação; histograma; gráfico ou carta de controle; e folha de verificação.

Para Barbosa (2000, p. 1), "as sete ferramentas do controle da qualidade são recursos a serem utilizados na aplicação da metodologia de solução de problemas."

Alvarez (2001) menciona que as ferramentas da qualidade proporcionam o apoio necessário para coleta, classificação, análise e apresentação de dados, para a prevenção e a solução de problemas futuros.

\subsubsection{Fluxograma ou diagrama de processo}

Essa ferramenta é uma simples representação das etapas de um processo. É uma forma organizada de documentar todas as atividades executadas por uma pessoa, ou por uma equipe dentro de uma estação de trabalho, que envolva clientes e materiais (KRAJEWSKI; RITZMAN; MALHOTA, 2009).

Os diagramas de processo têm como objetivo listar de uma forma simples as etapas do processo, para uma breve visualização e entendimento (CORRÊA; CORRÊA, 2008).

Para Peinado e Graeml (2007), o fluxograma é uma ferramenta de grande utilidade, podendo apresentar as seguintes aplicações: aperfeiçoar o entendimento do processo, indicar 
TAVARES, P. A.; RAMOS, M. C.; PEÇANHA, A. da S. Aplicação das sete ferramentas da qualidade em uma empresa de recapagem de pneus no centro-oeste de Minas Gerais.

como deve ser realizado o trabalho e inventar um modelo de trabalho ou um regulamento de como proceder.

\subsubsection{Análise de Pareto}

A análise de Pareto ou gráfico de Pareto é uma ferramenta que classifica os problemas ou as causas por ordem de importância. Paladini (1997) descreve que são usados gráficos de barras na classificação dos problemas de um processo de acordo com sua importância.

Coraiola (2001) descreve as principais aplicações do gráfico de Pareto, sendo: encontrar problemas; encontrar as causas que operam em um defeito; aperfeiçoar a visão de uma ação; dar preferência para a ação; aprovar os resultados de melhoria; delinear as causas de maior significância, excluindo sua causa; separar em classes a ação; e apontar os elementos responsáveis pelos impactos de maior importância.

\subsubsection{Diagrama de causa ou efeito - Diagrama de Ishikawa}

Também conhecido como espinha de peixe, esta ferramenta é um diagrama que relaciona um efeito (problema) com sua causa. Na cabeça do peixe fica a descrição do problema. A partir do desenho que representaria a espinha dorsal do peixe, são acrescidas as ramificações onde estão descritas as possíveis causas para cada problema. Parte-se das causas que são consideradas gerais até chegar àquelas que são consideradas as raízes do problema (CORRÊA; CORRÊA, 2008).

Araújo (2001) acredita que o diagrama de causa ou efeito é uma ferramenta importante para os processos de planejamento, podendo favorecer no entendimento e na organização das ideias.

\subsubsection{Diagrama de dispersão ou correlação}

Esta ferramenta é utilizada para descobrir possíveis relações entre os problemas e o tempo, ou entre os problemas e suas possíveis causas.

Paladini (1997, p. 74) afirma que “os diagramas de dispersão resultam de simplificações efetuadas em procedimentos estatísticos usuais e são modelos que permitem rápido relacionamento entre as causas e os efeitos." 
TAVARES, P. A.; RAMOS, M. C.; PEÇANHA, A. da S. Aplicação das sete ferramentas da qualidade em uma empresa de recapagem de pneus no centro-oeste de Minas Gerais.

Caraiola (2001) aponta as seguintes utilizações dos diagramas de dispersão: considerar uma variável com outra e visualizar o que ocorre se uma sofrer alteração; apurar se há relacionamento entre as duas variáveis, ou se há possibilidade de relação de causa ou efeito; considerar a intensidade do relacionamento entre as duas variáveis, e fazer comparação da relação entre os dois efeitos.

\subsubsection{Histogramas}

Histograma é um gráfico de barras que apresenta as informações de uma maneira que possibilite a visualização da distribuição dos dados, possibilitando a percepção do valor central e da dispersão dos dados ao seu entorno (LIMA, 2006).

Para Krajewski, Ritzman e Malhotra (2009, p. 134), "um histograma resume os dados medidos em uma escala contínua, mostrando a distribuição de frequência de alguma característica de classe."

Dentre as aplicações do histograma, Coraiola (2001) aponta: examinar o número de produto que não está conforme; definir a dispersão dos valores de medidas em objetos; avaliar ações que necessitam ser corrigidas; e localizar e apontar por meio de número de unidade, por cada categoria.

\subsubsection{Gráfico ou Carta de Controle}

Peinado e Graeml (2007) relatam que gráficos de controle são usados para constatar se determinado processo está de acordo com os limites de controle, ou seja, se o processo está de acordo com o planejado.

Logo, os gráficos de controle ajudam na agilidade da tomada de decisão, uma vez que esse gráfico mostra, de forma clara, se um determinado processo está fora de controle.

Para Tachizawa e Scaico (1997), esse gráfico é usado quando é necessário monitorar o comportamento de um processo que está em andamento, para determinar se está sendo válido e/ou se há oportunidade de melhoria para o processo.

Segundo Corrêa e Corrêa (2008, p. 221), “o objetivo das cartas é o de manter o controle de um processo através do acompanhamento do comportamento de uma ou várias medidas importantes resultantes desse processo". 
TAVARES, P. A.; RAMOS, M. C.; PEÇANHA, A. da S. Aplicação das sete ferramentas da qualidade em uma empresa de recapagem de pneus no centro-oeste de Minas Gerais.

\subsubsection{Folhas de verificação}

Rotondaro (2002) relata que, em uma folha de verificação, é necessário constar vários itens, como: nome da empresa, produto examinado, período da coleta, nome do coletador, data, identificação do lote, ou seja, deve possuir todas as informações necessárias para a análise do processo.

Paladini (1997, p. 67) complementa que "elas são estruturadas de acordo com as necessidades específicas de seus usuários e, por isso, apresentam extrema flexibilidade de elaboração, utilização e interpretação.”

\section{MATERIAIS E MÉTODOS}

Este trabalho pode ser classificado, quanto à sua natureza, como pesquisa aplicada pela aplicação prática de conhecimentos. Quantos aos objetivos, o trabalho tem caráter exploratório, pois, de acordo com Gil (1999), é desenvolvido com a finalidade de proporcionar uma visão total, do tipo de aproximação, acerca de um determinado fato, em que será utilizado. Quanto à abordagem, a pesquisa é classificada como pesquisa qualitativa e o método de pesquisa utilizado foi o estudo de caso, que consiste em aprofundar de forma exaustiva um ou poucos objetos, de tal forma que se consiga um conhecimento amplo e detalhado do objeto de pesquisa.

\subsection{Objeto de pesquisa}

O objeto de pesquisa deste trabalho foi uma empresa de recapagem de pneus no Centro-Oeste de Minas Gerais. No intuito de garantir a confidencialidade de seus dados, ela passa a ser denominada como empresa X. Foi fundada em 2006 e possui atualmente 20 funcionários. A empresa estudada pertence a um grupo conceituado, com atuação em Minas Gerais e São Paulo. A empresa X é parceira de um grande fornecedor que possui uma tecnologia avançada de reformas de pneus.

\subsection{Coleta de dados}


TAVARES, P. A.; RAMOS, M. C.; PEÇANHA, A. da S. Aplicação das sete ferramentas da qualidade em uma empresa de recapagem de pneus no centro-oeste de Minas Gerais.

Segundo Oliveira (1999), a coleta de dados é a etapa da pesquisa que exige mais tempo, por isso o pesquisador precisa ter paciência e esforço, além do cuidado e atenção para coletar e registrar os dados.

A coleta de dados foi realizada por meio de análise de documentos da empresa, tais como planilhas de controle de produção e da qualidade interna. Os dados coletados para a aplicação das sete ferramentas da qualidade correspondem ao período de janeiro de 2011 a agosto de 2012, divididos por ano.

\subsection{Interpretação de dados}

Para a interpretação dos dados pesquisados, foram utilizadas planilhas do Excel e os dados coletados foram registrados para avaliação do processo de produção. Também foi utilizado o programa Minitab, ferramenta de grande auxílio para a interpretação dos dados, no qual foram elaborados os gráficos de controle, os histogramas e os diagramas.

\section{RESULTADOS E DISCUSSÃO}

O processo de recapagem de pneus possui várias etapas em seu processo, sendo de suma importância conhecer cada etapa, avaliar as falhas que envolvem cada uma delas, adotar medidas que minimizem os impactos causados por elas, no sentido de corrigi-las, evitando assim os prejuízos que acarretam para o processo produtivo.

\subsection{Aplicação das sete ferramentas da qualidade}

Após a análise das etapas do processo produtivo da empresa, foram aplicadas as sete ferramentas da qualidade, visando analisar o processo.

Elas foram aplicadas, obedecendo à seguinte sequência: fluxograma, folha de verificação, diagrama de causa ou efeito, gráfico de Pareto, diagrama de dispersão, histograma e gráficos de controle.

\subsubsection{Fluxograma}

A FIG. 1 apresenta de forma simplificada todas as etapas que compõem o processo de recapagem.

Esse fluxograma deixa claro que as etapas de inspeção inicial e inspeção final são de 
TAVARES, P. A.; RAMOS, M. C.; PEÇANHA, A. da S. Aplicação das sete ferramentas da qualidade em uma empresa de recapagem de pneus no centro-oeste de Minas Gerais.

extrema importância e merecem uma atenção redobrada, por se tratarem de etapas decisórias.

Figura 1 - Etapas do processo de recapagem

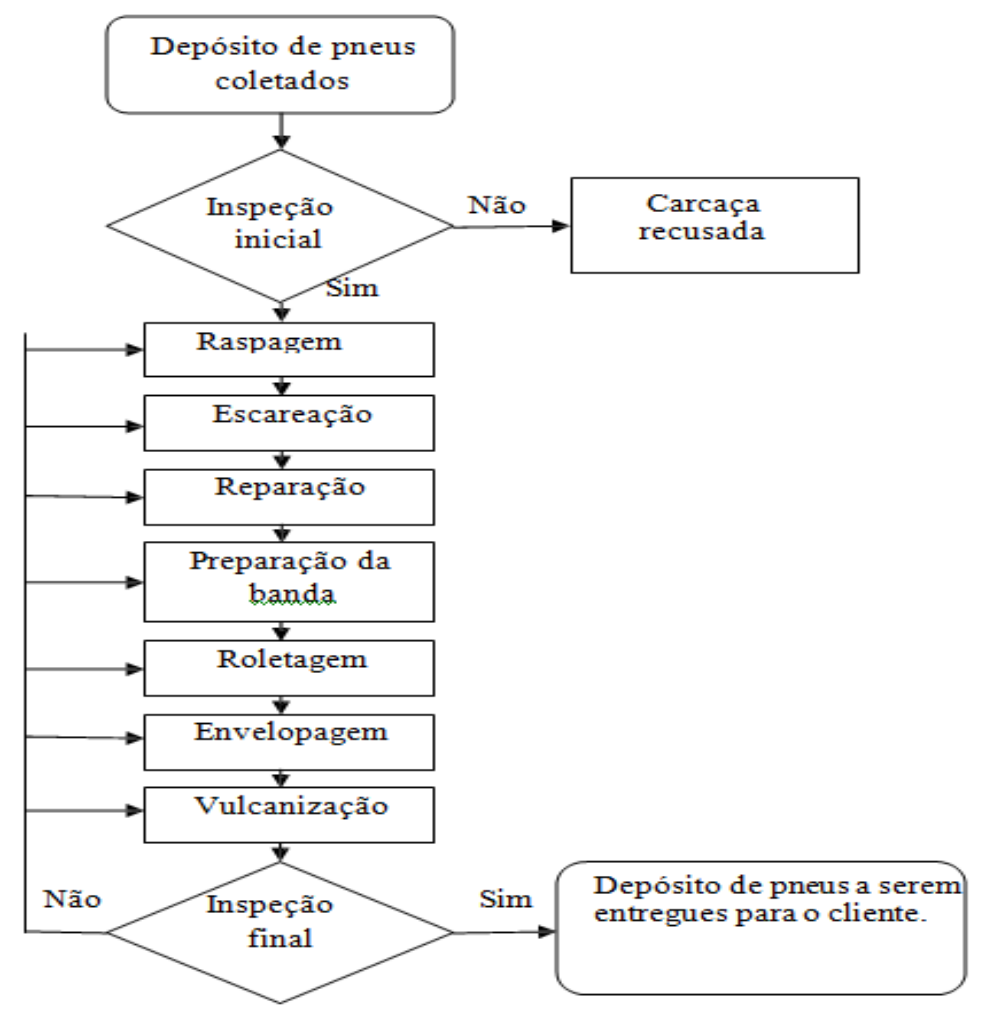

Fonte: Dados da pesquisa, 2012.

\subsubsection{Folha de Verificação}

Esta ferramenta representou de forma simples a frequência dos problemas ocorridos no período de janeiro a dezembro de 2011 e de janeiro a agosto de 2012.

A TAB. 1 demonstrou que a etapa de escareação apresenta maior número de defeitos, e apresenta uma diferença significativa em relação à segunda etapa, que é a reparação. $\mathrm{O}$ número de defeitos é maior que o número de pneus rejeitados. É possível perceber que a escareação é a etapa crítica do processo.

A TAB. 2 demonstra que, novamente, o maior número de defeitos foi percebido na escareação e, em seguida, a gomagem referente à etapa de reparação. Nesse período, o cozimento (etapa de vulcanização) apresentou menor defeito. A proporção de número de defeitos com pneus rejeitados entre 2011 e 2012 apresenta porcentagens relativamente iguais. Como no ano anterior, a etapa crítica do processo foi a escareação. 
TAVARES, P. A.; RAMOS, M. C.; PEÇANHA, A. da S. Aplicação das sete ferramentas da qualidade em uma empresa de recapagem de pneus no centro-oeste de Minas Gerais.

Tabela 1 - Folha de Verificação ano 2011

ESTÁGIO DE FABRICAÇãO: inspeção final PERÍODO: ANO 2011

Tipo de defeito: colagem, cozimento, escareação, gomagem, inspeção final, inspeção inicial, preparação, roletagem

Seção: Recapagem

\begin{tabular}{|c|c|c|}
\hline \multicolumn{2}{|l|}{ Total inspecionado: 8466} & Inspetor: Leonardo \\
\hline \multicolumn{3}{|c|}{ Obs.: todos os itens produzidos foram inspecionados } \\
\hline DEFEITO - ETAPA & MARCA & SUB-TOTAL \\
\hline Colagem - reparação & I & 1 \\
\hline Cozimento - vulcanização & IIIIIIIIIII & 10 \\
\hline Escareação & IIIIIIIIIIIIIIIIIIIIIIIIIIIIIIII & 32 \\
\hline Gomagem - reparação & IIIIIIIIIIIIIIII & 14 \\
\hline Inspeção final & IIII & 4 \\
\hline Inspeção inicial & IIIIIIIIIIII & 11 \\
\hline Prep. BDR & III & 3 \\
\hline Roletagem & III & 3 \\
\hline & TOTAL & 78 \\
\hline TOTAL REJEITADO & IIIIIIIIIIIIIIIIIIIIIIIIIIIIIIIIIIIIIIIIIIIIIIIIIIII & 56 \\
\hline
\end{tabular}

Fonte: Dados da Pesquisa, 2012.

Tabela 2 - Folha de Verificação do ano de 2012

\begin{tabular}{|c|c|c|}
\hline \multicolumn{2}{|c|}{ ESTÁGIO DE FABRICAÇÃO: inspeção final } & PERÍODO: JANEIRO A AGOSTO 2012 \\
\hline \multicolumn{2}{|c|}{$\begin{array}{l}\text { Tipo de defeito: cozimento, escareação, gomagem, inspeção inicial, } \\
\text { manchão }\end{array}$} & Seção: Recapagem \\
\hline \multicolumn{2}{|l|}{ Total inspecionado: 6172} & Inspetor: Leonardo \\
\hline \multicolumn{3}{|c|}{ Obs.: todos os itens produzidos foram inspecionados } \\
\hline DEFEITO - ETAPA & MARCA & SUBTOTAL \\
\hline Cozimento - vulcanização & I & 1 \\
\hline Escareação & IIIIIIIIIIIIIIIII & 16 \\
\hline Gomagem - reparação & IIIIIIIIIIIIII & 13 \\
\hline Inspeção inicial & III & 3 \\
\hline Manchão - reparação & IIII & 4 \\
\hline \multicolumn{2}{|r|}{ TOTAL } & 37 \\
\hline TOTAL REJEITADO & IIIIIIIIIIIIIIIIIIIIIIIIIIIIIII & 29 \\
\hline
\end{tabular}

Fonte: Dados da Pesquisa, 2012

\subsubsection{Gráfico de Pareto}

No caso em estudo, foram classificados os problemas ocorridos durante o período de 
TAVARES, P. A.; RAMOS, M. C.; PEÇANHA, A. da S. Aplicação das sete ferramentas da qualidade em uma empresa de recapagem de pneus no centro-oeste de Minas Gerais.

janeiro a dezembro de 2011, no GRAF. 1 e de janeiro a agosto de 2012 no GRAF. 2.

Gráfico 1 - Gráfico de Pareto no período compreendido entre janeiro a dezembro de 2011

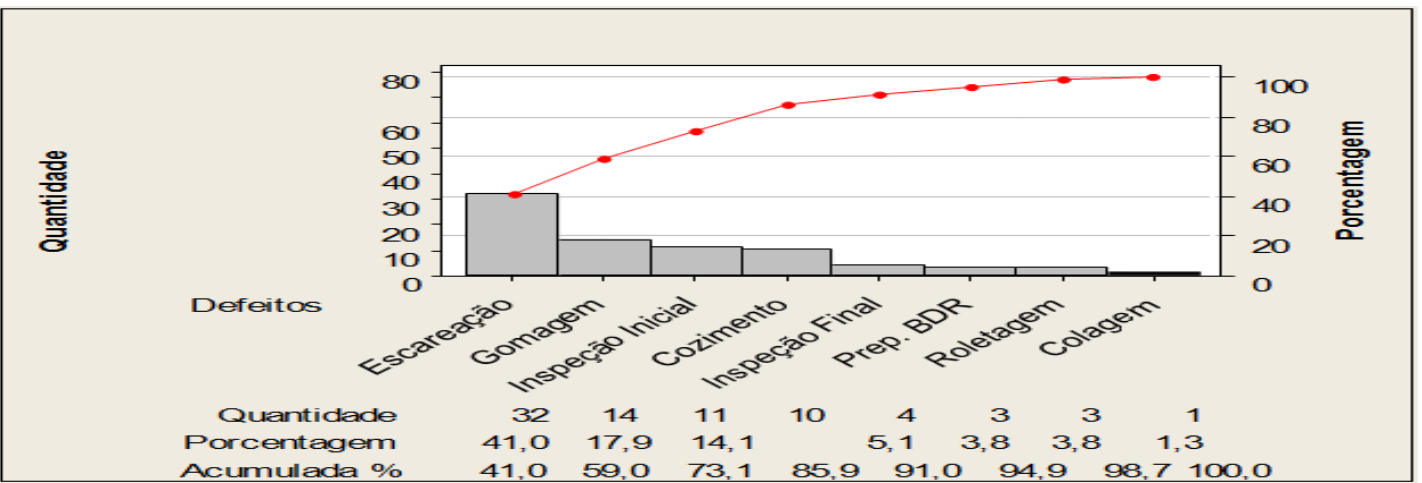

Fonte: Dados da pesquisa, 2012.

O GRAF. 1 mostra que a escareação apresenta maior frequência de defeitos, seguida da gomagem (realizada na reparação), inspeção inicial, cozimento (realizado na vulcanização) e inspeção final. A colagem (realizada na reparação) apresenta menor frequência de defeitos, e posteriormente a roletagem e preparação de banda. As etapas de escareação e reparação apresentam 59\% de frequência, sendo a escareação a etapa crítica.

Gráfico 2 - Gráfico de Pareto no período compreendido entre janeiro a agosto de 2012

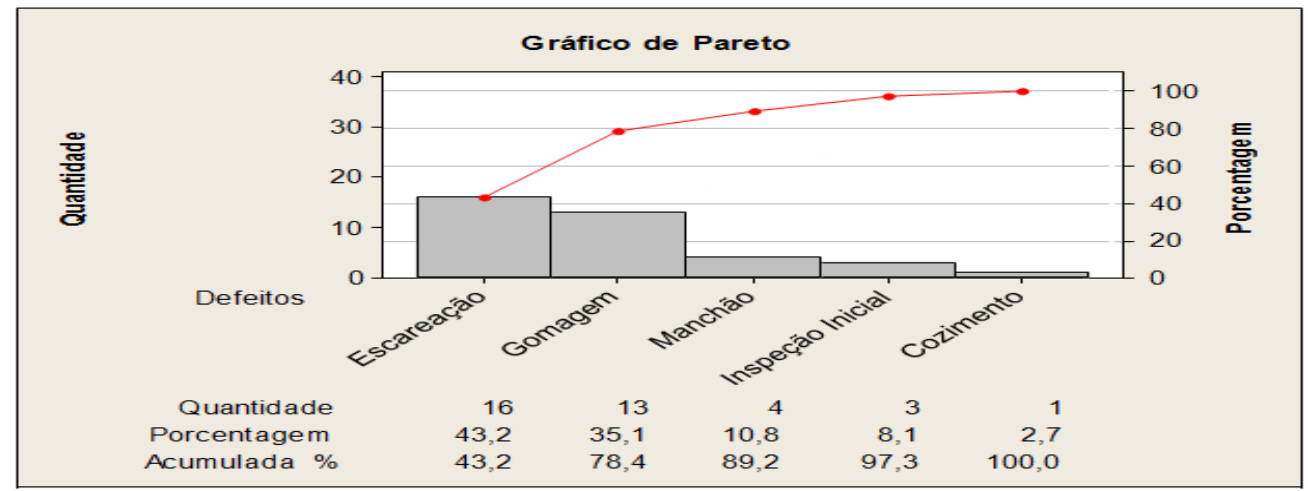

Fonte: Dados da pesquisa, 2012.

No GRAF. 2, a escareação apresenta novamente maior frequência de defeitos, seguida da gomagem (realizada na reparação). O cozimento (realizado na vulcanização) apresenta menor frequência. A escareação e a gomagem (reparação) apresentam uma alta porcentagem de frequência, atingindo 78,4\%. A escareação continua sendo a etapa crítica do processo.

\subsubsection{Diagrama de dispersão}


TAVARES, P. A.; RAMOS, M. C.; PEÇANHA, A. da S. Aplicação das sete ferramentas da qualidade em uma empresa de recapagem de pneus no centro-oeste de Minas Gerais.

A aplicação desta ferramenta permitiu verificar se existem possíveis relações entre a produção e os defeitos. A seguir, os diagramas de dispersão realizados na empresa relacionam a quantidade de pneus produzidos com a quantidade de pneus rejeitados.

O GRAF. 3 mostra os dados referentes ao ano de 2011 e o GRAF. 4 dados de 2012.

Gráfico 3 - Diagrama de dispersão no período de janeiro a dezembro de 2011

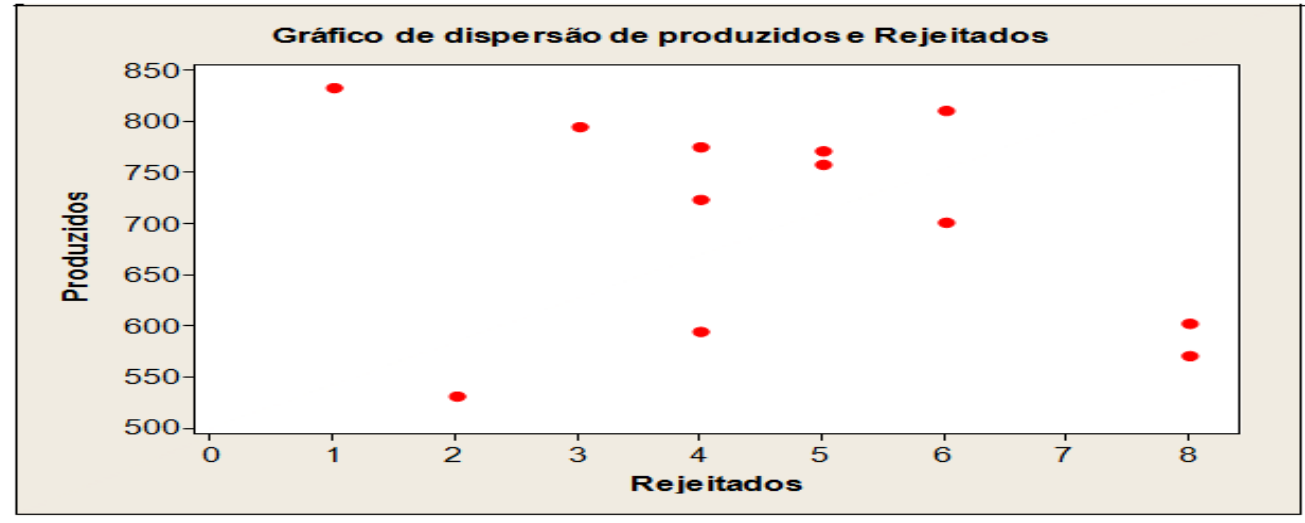

Fonte: Dados da pesquisa, 2012.

O GRAF. 3 permite observar que os pontos ficaram bem distribuídos e que não existe uma forte relação entre o número de produção e o número de pneus rejeitados. Pode-se observar que em um mês foram produzidos em média 830 pneus, sendo que apenas um foi rejeitado; já em outro mês foram produzidos 570 pneus e oito foram rejeitados.

Gráfico 4 - Diagrama de dispersão no período de janeiro a agosto de 2012

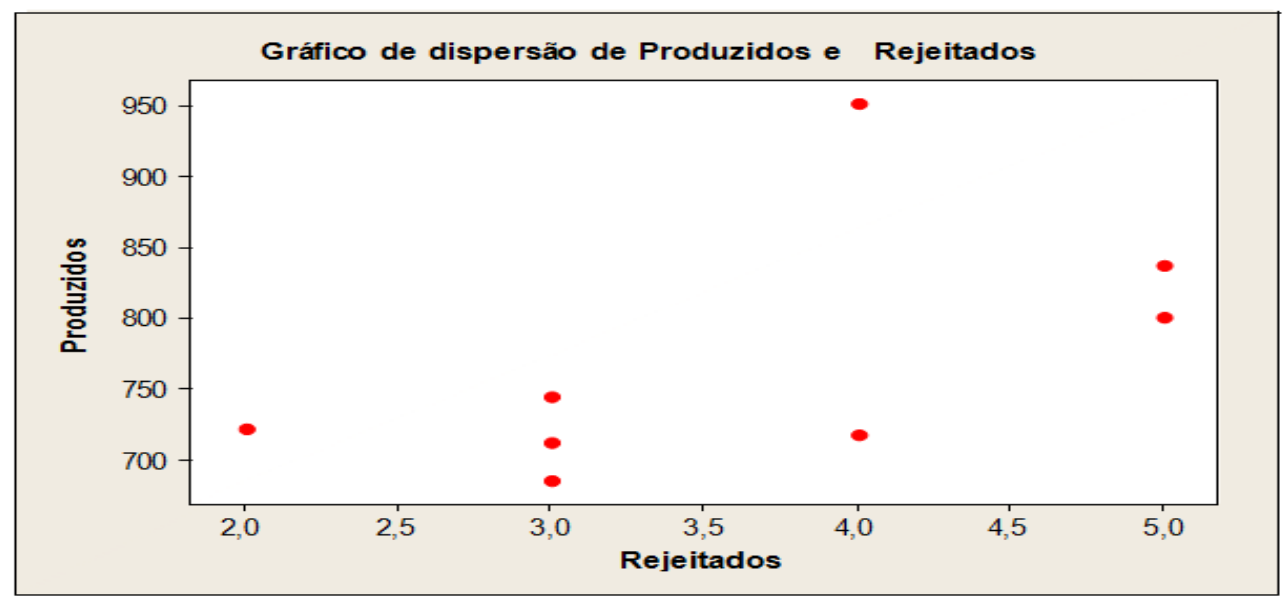

Fonte: Dados da pesquisa, 2012.

O GRAF. 4 demonstra que os pontos do gráfico do ano de 2011 ficaram mais distribuídos que os pontos do gráfico de do ano de 2012. Novamente percebe-se que não existe uma forte relação entre a quantidade de pneus produzidos e a quantidade de pneus rejeitados. Num mês foram produzidos 950 pneus, sendo 4 rejeitados, e em outro mês foram 
TAVARES, P. A.; RAMOS, M. C.; PEÇANHA, A. da S. Aplicação das sete ferramentas da qualidade em uma empresa de recapagem de pneus no centro-oeste de Minas Gerais.

produzidos 840 pneus, sendo 5 rejeitados.

\subsubsection{Histograma}

O GRAF. 5 mostra o histograma com a frequência de erros nas etapas do processo de recapagem da empresa X, referente ao ano de 2011 e o GRAF. 6 mostra o histograma correspondente ao ano de 2012

Gráfico 5 - Histograma no período compreendido entre janeiro a dezembro de 2011

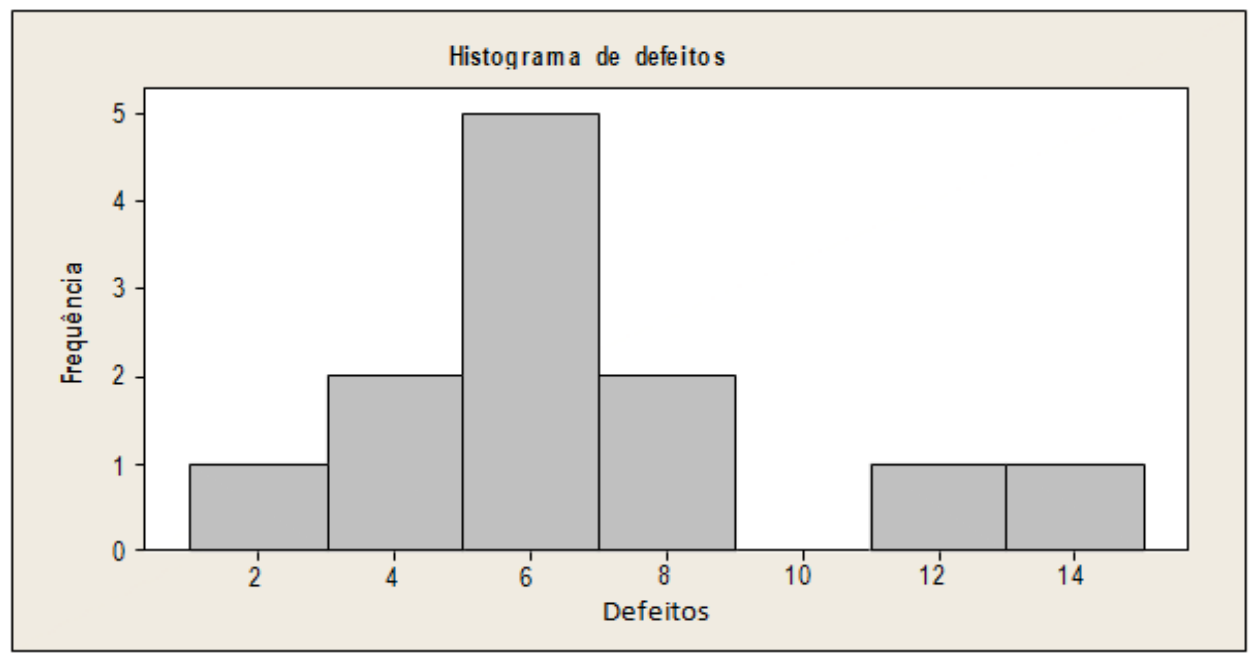

Fonte: Dados da pesquisa, 2012.

Pode-se observar que o histograma apresenta assimetria positiva, devido aos maiores índices de defeitos (entre 11 a 15) estarem presentes na classe 1, sendo a classe de menor frequência. Os defeitos de maior frequência estão relacionados às etapas de escareação e reparação.

Gráfico 6 - Histograma no período compreendido entre janeiro a agosto de 2012

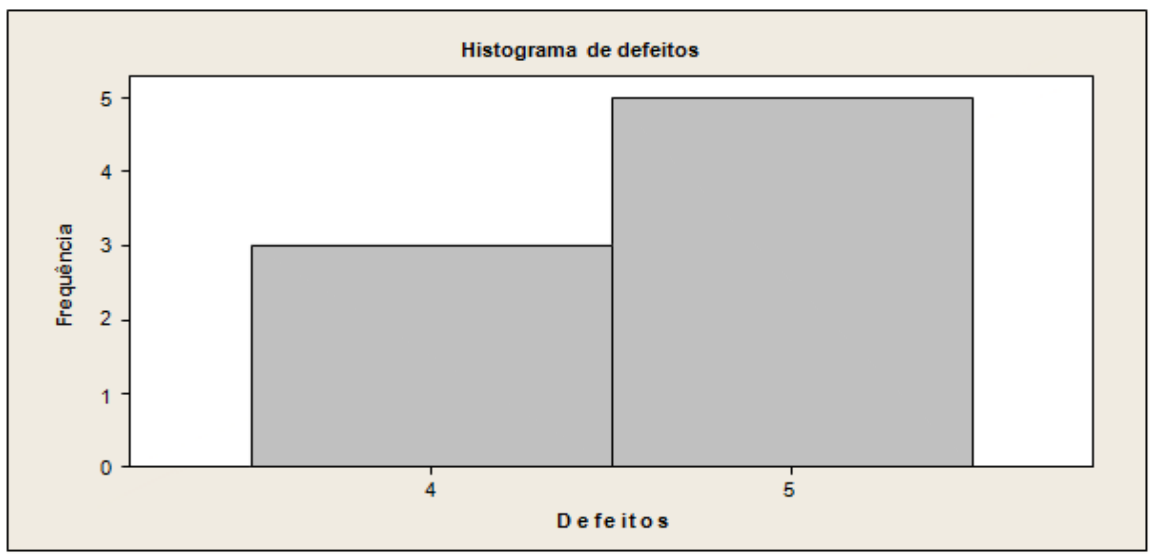

Fonte: Dados da pesquisa, 2012. 
TAVARES, P. A.; RAMOS, M. C.; PEÇANHA, A. da S. Aplicação das sete ferramentas da qualidade em uma empresa de recapagem de pneus no centro-oeste de Minas Gerais.

Neste histograma, pode-se observar que não houve uma assimetria positiva, sendo que o maior número de defeitos (5) localiza-se na classe de maior frequência, sendo a classe 5 . Nota-se que os números de defeitos no período de 2012 foram distribuídos em apenas duas quantidades. Novamente os defeitos de maior frequência estão relacionados às etapas de escareação e reparação.

\subsubsection{Gráfico de controle}

Os gráficos de controle foram usados para monitorar o controle de processos, sendo que o GRAF. 7 demonstra o controle de processo da empresa X, referente ao ano de 2011. No GRAF. 8, são demonstrados os dados correspondentes ao ano de 2012.

Gráfico 7 - Gráfico de controle no período de janeiro a dezembro de 2011

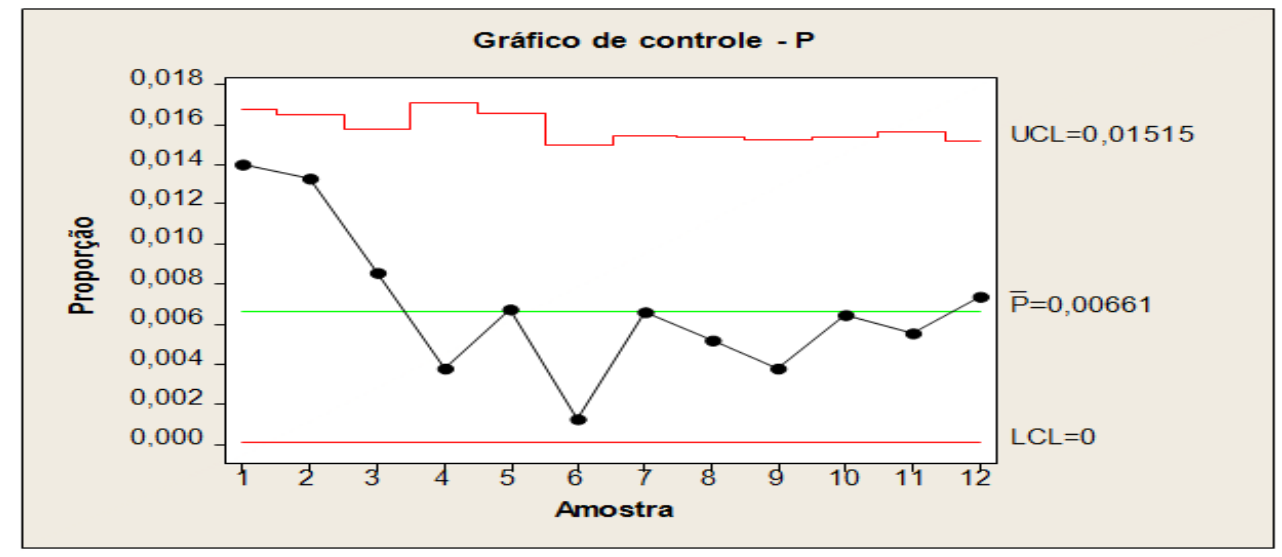

Fonte: Dados da pesquisa, 2012.

O GRAF. 7 apresenta alguns pontos que estão fora do controle, ultrapassando a linha central - linha de controle. As etapas do processo que estão fora de controle são a escareação e a reparação. 
TAVARES, P. A.; RAMOS, M. C.; PEÇANHA, A. da S. Aplicação das sete ferramentas da qualidade em uma empresa de recapagem de pneus no centro-oeste de Minas Gerais.

Gráfico 8 - Gráfico de controle no período de janeiro a agosto de 2012

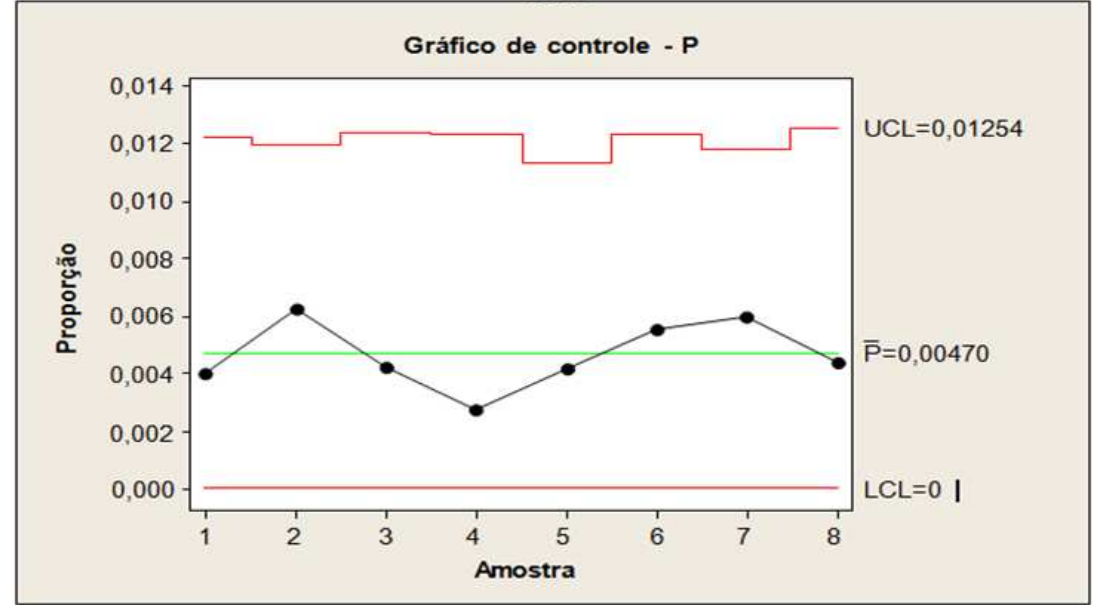

Fonte: Dados da pesquisa, 2012.

O GRAF. 8 demonstra que os pontos deste controle estão distribuídos aleatoriamente, mas que existem alguns pontos que estão ultrapassando a linha de controle - linha central. Novamente a escareação e a reparação são as etapas que estão fora de controle.

\subsubsection{Diagrama de causa ou efeito}

A aplicação desta ferramenta foi de grande importância, possibilitando perceber as possíveis causas para os problemas. A FIG. 2 mostra o diagrama de causa e efeito realizado na empresa $X$.

Figura 2 - Diagrama de causa ou efeito

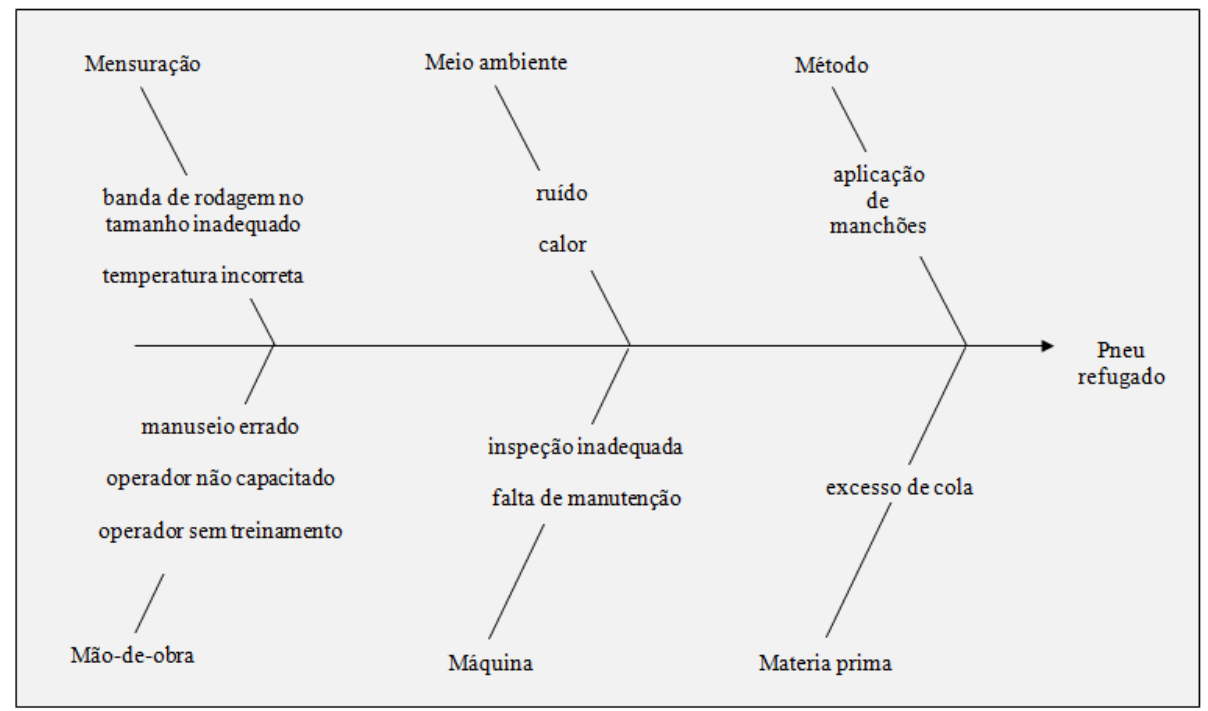

Fonte: Dados da pesquisa, 2012. 
TAVARES, P. A.; RAMOS, M. C.; PEÇANHA, A. da S. Aplicação das sete ferramentas da qualidade em uma empresa de recapagem de pneus no centro-oeste de Minas Gerais.

A aplicação destas ferramentas mostrou que a escareação é a etapa crítica do processo, sendo a etapa que apresenta maior frequência de defeitos. Ela está relacionada com a mão de obra. Desta forma, as possíveis causas para os problemas são mão de obra e, em seguida, máquina e meio ambiente, que também estão relacionados diretamente com a mão de obra.

\subsection{Sugestões de ações para melhorias}

Com a aplicação destas ferramentas, foi possível perceber que a escareação e a reparação são as etapas que apresentam maior frequência de defeitos, assim essas etapas devem ser priorizadas. Por se tratar de etapas que dependem diretamente de mão de obra, deve-se tomar medidas no intuito de capacitar os empregados que estão envolvidos diretamente nessas etapas.

Entre as medidas que podem ser adotadas para corrigir as falhas percebidas ao longo desse estudo, vale ressaltar:

Realizar periodicamente treinamentos e reciclagens dos funcionários, visando capacitá-los, no intuito de descobrir com rapidez as anormalidades no processo, ter melhor controle da situação e capacidade de recuperação. Os colaboradores precisam estar conscientes de que é muito importante cumprir todas as regras estabelecidas, para assegurar a qualidade.

Realizar um programa de manutenção mais eficiente e estratégico, em que seja realizada uma rigorosa manutenção dos equipamentos envolvidos no processo.

Melhorar a iluminação, instalando lâmpadas mais eficientes, principalmente na etapa de escareação, local que necessita de uma melhor iluminação.

\section{CONCLUSÃO}

A aplicação das sete ferramentas da qualidade possibilitou maior percepção das etapas dos processos que necessitam de melhorias que garantam melhores resultados para a empresa e seus clientes finais.

As sugestões descritas neste trabalho apontam alternativas para os erros percebidos na busca pela melhoria na produção e racionalização dos processos com impacto no resultado final, tornando-o mais produtivo, eficiente e eficaz. 
TAVARES, P. A.; RAMOS, M. C.; PEÇANHA, A. da S. Aplicação das sete ferramentas da qualidade em uma empresa de recapagem de pneus no centro-oeste de Minas Gerais.

\section{REFERÊNCIAS}

ALVAREZ, M. E. B. Administração da qualidade e da produtividade: abordagens do processo administrativo. São Paulo: Atlas, 2001.

ARAÚJO, L. C. G. de. Organização, sistemas e métodos e as modernas ferramentas de gestão organizacional: arquitetura, benchmarking, empowerment, gestão pela qualidade total, reengenharia. São Paulo: Atlas, 2001.

BARBOSA, E. F. Sete ferramentas do controle de qualidade: gerência da qualidade total na educação. Belo Horizonte: Fundação Cristiano Ottoni/UFMG, 2000.

CAMPOS, V. F. TQC: controle da qualidade total. Belo Horizonte: Fundação Cristiano Ottoni/UFMG, 1992.

CORAIOLA, J. A. Gerenciamento da rotina: uma metodologia das ferramentas da qualidade numa disciplina específica do curso de tecnologia em eletrotécnica do Cefet - PR. 2001. Dissertação (Mestrado em Engenharia de Produção e Sistemas) - Universidade Federal de Santa Catarina, Florianópolis, 2001.

CORRÊA, H. L.; CORRÊA, C. A. Administração de produção e operações: uma abordagem estratégica. São Paulo: Atlas, 2008.

GAITHER, N.; FRAZIER, G. Administração da produção e operações. São Paulo: Thomson Learning, 2007.

GIL, A. C. Métodos e técnicas de pesquisa social. São Paulo: Atlas, 1999.

GIANESI, I. G. N.; CORRÊA, H. L. Administração estratégica de serviços: operações para a satisfação do cliente. São Paulo: Atlas, 1996.

JURAN, J. M.; GRYNA, F. M. Controle da qualidade. São Paulo: Makron Books, 1991.

KRAJEWSKI, L. J.; RITZMAN, L.; MALHOTRA, M. Administração de produção e operações. São Paulo: Pearson Prentice Hall, 2009.

LIMA, R. A. Como a relação entre clientes e fornecedores internos de uma organização pode contribuir para a garantia da qualidade: o caso de uma empresa automobilística. 2006. Monografia (Graduação em Engenharia de Produção)-Universidade Federal de Ouro Preto, Ouro Preto, 2006.

LONGO, R. M. J. Gestão da qualidade: evolução histórica, conceitos básicos e aplicação na educação. In: SEMINÁRIO GESTÃO DA QUALIDADE NA EDUCAÇÃO: em busca da excelência. Anais... Brasília: IPEA, 1996.

MIGUEL, P. A. C. Qualidade: enfoque e ferramentas. São Paulo: Artliber, 2001.

OLIVEIRA, O. J. et al. Gestão da qualidade: tópicos avançados. São Paulo: Thomson Learning, 2006. 
TAVARES, P. A.; RAMOS, M. C.; PEÇANHA, A. da S. Aplicação das sete ferramentas da qualidade em uma empresa de recapagem de pneus no centro-oeste de Minas Gerais.

OLIVEIRA, S. L. de. Tratado de metodologia científica: projetos de pesquisas, TGI, TCC, monografias, dissertações e teses. São Paulo: Pioneira, 1999.

PALADINI, E. P. Gestão da qualidade no processo: a qualidade na produção de bens e serviços. São Paulo: Atlas, 1995.

Qualidade total na prática: implantação e avaliação de sistemas de qualidade total. São Paulo: Atlas, 1997.

PEINADO, J.; GRAEML, A. R. Administração da produção: operações industriais e de serviços. Curitiba: UnicenP, 2007.

ROTONDARO, R. G. Método básico: uma visão geral. In: Seis Sigmas: estratégia gerencial para melhoria de processo, produto e serviços. São Paulo: Atlas, 2002.

TACHIZAWA, T.; SCAICO, O. Organização flexível: qualidade na gestão por processos. São Paulo: Atlas, 1997. 